\title{
Functional Significance of miRNA-149 in Lung Cancer: Can it be Utilized as a Potential Biomarker or a Therapeutic Target?
}

\author{
Chauhan SJ, Thyagarajan A and Sahu RP* \\ Department of Pharmacology and Toxicology, Boonshoft \\ School of Medicine, Wright State University, Dayton, USA \\ *Corresponding author: Sahu RP, Department of \\ Pharmacology and Toxicology Boonshoft School of \\ Medicine, Wright State University 3640 Colonel Glenn \\ Hwy, Dayton, USA
}

Received: May 21, 2020; Accepted: June 10, 2020; Published: June 17, 2020

\begin{abstract}
Accumulating evidence has documented the significance of miR-149 as a promising tumor-suppressive non-coding RNA that plays critical roles in regulating genes involved in cancer growth and metastasis. Notably, the ability of miR-149 to be utilized as a potential biomarker in the diagnosis/prognosis or a therapeutic target has also been explored using various cellular and preclinical models, as well as in clinical settings of lung cancer. While the applicability of miR-149 in assessing tumor progression has been suggested, its potential in predicting treatment outcomes is needed to be verified in diverse settings of lung cancer patients. The current review presents an overview of the functional significance of miR-149 with ongoing challenges in non-small cell lung cancer.
\end{abstract}

Keywords: Lung cancer; miR-149; Cancer therapy

\section{Abbreviations}

NSCLC: Non-Small Cell Lung Cancer; SCLC: Small Cell Lung Cancer; miRs: MicroRNAs; UTR: 3'untranslated region; SOX2: SRY-Box Transcription Factor 2; GSK3a - Glycogen Synthase Kinase-3 $\alpha$; JunB - AP-1 Transcription Factor Subunit AKT1 Protein kinase B; PARP-2: Poly [ADP-ribose] polymerase 2; GIT1: G-protein-Coupled Receptor Kinase Interacting Protein 1; ErbB3: Receptor Tyrosine-Protein Kinase-3; Rapla: Ras-Related Protein Rap-1A Rap2b - Ras-Related Protein Rap-2B; NDST1: Bifunctional Heparan Sulfate N-Deacetylase/N-Sulfotransferase; 1 PPM1F: Protein Phosphatase Mg2+/Mn2+ Dependent 1F; ZBTB2: Zinc finger and BTB domain containing 2 Wnt-1: Proto-oncogene protein Wnt-1; E2F1: Transcription Factor E2F1; EMT: Epithelialto-Mesenchymal Transition; ECM - Extracellular Matrix; MMPs: Matrix Metalloproteinases; MMP-2: Matrix Metalloproteinase -2; POSTN: Periostin; VCAN: Versican; FOXM1: Forkhead Box Protein M1; LncRNAs: Long non-coding RNAs; HNF1A-AS1: Hepatocyte Nuclear Factor-1 homeobox A (HNF1A)-antisense RNA 1 (AS1); Cdk6: Cyclin-Dependent Kinase 6; PCAT-1: Prostate CancerAssociated ncRNA Transcripts 1; LRIG2: Leucine-Rich Repeats and Immunoglobulin like domains 2; BcYrn1: Brain cytoplasmic RNA 1; LINC00472: Long Intergenic Non-Protein-Coding RNA 472; CSCs: Cancer stem cells; LINC00460: long intergenic non-proteincoding; RNA 460 SNPs: Single Nucleotide Polymorphisms; TOP1: Topoisomerases 1 pathway

\section{Introduction}

While prostate and breast cancers were estimated the most prevalent cancers in 2019 affecting both males and females, respectively, lung cancer was regarded as the second most predominant cancer accounting for up to $13 \%$ of all cancer cases diagnosed in the United States [1-6]. Also, lung cancer remains the leading cause of cancer-related deaths affecting both men and women with mortality rates of $23 \%$ and $24 \%$, respectively [1-10]. Notably, the two main types of lung cancer, namely Small Cell Lung Carcinoma (SCLC), and Non-Small Cell Lung Carcinoma (NSCLC) majorly differ in their site of origin, and an overall prevalence of cancer cases (15\% versus $85 \%)$, respectively [11-13]. Based upon their site of origin, NSCLC is further divided into 3 subtypes, namely, adenocarcinoma, squamous cell carcinoma, and large cell carcinoma [11-13]. While the cellular, molecular, and genetic modifications underlying the pathogenesis of lung cancer are becoming apparent, the exact mechanism governing the development of normal respiratory epithelium to neoplastic transformation is still not very well defined. This restricts the ability for early diagnosis or prognosis, as well as in overcoming chemoresistance induction of this cancer type, which continues to be the main wellness and socio-economic issues [7-9,14,15].

Importantly, several risk factors have been identified to be associated with lung carcinogenesis including environmental factors, and pollutants such as cigarette smoking, arsenic exposure, etc., as well as genetic modifications $[7,8]$. In the United States, cigarette smoking continues to be the major risk factor for lung cancer, which accounts for $80 \%$ to $90 \%$ of lung cancer deaths [10]. Several of these risk factors can contribute to lung carcinogenesis via their abilities to induce alterations in cellular signaling pathways, and their regulations, etc. Thus, to understand the whole cellular events involved in lung tumorigenesis has been complex and challenging, which necessitates the identification of biomarkers to aid in the diagnosis and/or prognosis. Of significance, cancer-specific microRNA expression signatures have been explored to understand several clinicopathological variables including tumor stages and metastasis, disease reoccurrence and treatment resistance, as well as patient survival [16-29].

MicroRNAs (miRs) are conserved single-stranded non-coding RNA molecules comprising of 19-22 nucleotides, which play important roles in essentially all the biological and pathophysiological 
processes including cancer [16-17]. The common biogenesis of miRs is a multi-step process. This involves various RNAse and RNA polymerase enzymes such as Drosha and Dicer, as well as helicase resulting in the conversion of primary miRs to precursor miRs, and finally to mature miRs [18-20]. miRs regulate gene expression via forming base pairing to the target mRNAs sequences within the 3'Untranslated Region (UTR), which results in either translation repression, direct mRNA degradation, or miR-mediated mRNA degradation [21-22]. Notably, several miRs have been identified to be expressed endogenously in any given cancer types, which can target hundreds of miRs. Therefore, miRs' expression signatures were primarily utilized to characterize the highly up- and down-regulated miRs, to then determine their mechanisms in cancer growth and metastasis and efficacy of cancer therapies.

To date, miRs are aberrantly expressed in human malignancies, and their up- or downregulation has been implicated to either promote or suppress cancer growth [23-25]. Importantly, genetic and epigenetic alterations have also been demonstrated to dysregulate the functions of miRs [26-47]. Contrary to most of the miRs that have been reported for their oncogenic effects, miR-149 has been identified to play dual roles (i.e., oncogenic and tumor-suppressive) depending upon the types of cancer. Of note, due to their translational significance, miRs including miR-149 have been explored for their therapeutic implications as well as biomarkers applicability in cancer models including lung cancer. In this review, we will discuss the functional significance of miR-149 as a promising therapeutic target and a potential biomarker in lung cancer.

miR-149 has been identified to be located on chromosomes 2 at 2 q37.3 [30]. Multiple studies have demonstrated that miR-149 plays critical roles in regulating various activities of tumor cells including cell proliferation and apoptosis. Notably, in glioblastoma multiforme, melanoma, prostate, and liver cancer models, miR149 plays oncogenic roles, which are mediated by the upregulation of its target genes such as SRY-Box Transcription Factor 2 (SOX2), Glycogen synthase kinase-3a (GSK3- $\alpha$ ), AP-1 transcription factor subunit (JunB), etc. $[31,33,36]$. However, in other cancer models such as neuroblastoma, hepatic, renal cell, breast, gastric, and pancreatic carcinomas, miR-149 functions as a regulator of tumor progression. This miR-149-induced effect was mediated via its ability to downregulate the target genes such as protein kinase B (AKT1), Poly [ADP-ribose] polymerase 2 (PARP-2), G-Protein-Coupled Receptor Kinase Interacting Protein 1 (GIT1), receptor tyrosine-protein kinase-3 (ErbB3), Ras-Related Protein Rap-1A (Rapla), Ras-Related Protein Rap-1B (Rap2b), bifunctional heparan sulfate N-deacetylase/ $\mathrm{N}$-sulfotransferase 1 (NDST1), protein phosphatase $\mathrm{Mg} 2+/ \mathrm{Mn} 2+$ dependent $1 \mathrm{~F}$ (PPM1F), zinc finger and BTB domain containing 2 (ZBTB2), proto- oncogene protein Wnt-1 (Wnt-1), transcription factor E2F1 (E2F1), etc. [31-41]. Particularly, in lung cancer models, miR-149 has been shown to function as a tumor suppressor via its ability to induce apoptosis $[42,43]$

\section{miR-149 as a therapeutic target}

In most cases, the cancer-related deaths occur because patients are usually diagnosed at an advanced and/or metastatic stage of the disease $[48,49]$. Therefore, the exploration of the link between tumor cells and its microenvironment is necessary to understand the underlying mechanisms of cancer metastasis to improve the treatment outcomes. In the initiation phase of metastasis, cancer progression requires the invasive potential of primary tumor cells, which is enabled through the Epithelial-To-Mesenchymal Transition (EMT). This process is characterized by the loss of E- cadherin mediated cell adhesion, and an increase in cell motility, which facilitates tumor invasion and metastasis to distant organs [48-50]. Importantly, the Extracellular Matrix (ECM) has been shown to be crucial in tumor tissue formation via its ability to regulate tumor cell adhesion, proliferation, differentiation, invasion, and migration [51,52]. Notably, several ECM components and proteins including matrix metalloproteinases (MMPs) such as MMP-1, MMP- 2, MMP9, and proteins such as Periostin (POSTN), and Versican (VCAN) are associated with the induction of EMT process and involved in favoring the metastatic potential of cancer cells [51, 53-57].

Since highly aggressive metastatic cancers such as colon, kidney, breast, and sarcoma often spread to the lungs, multiple studies have explored the significance of miRs to delineate the mechanism between lung cancer metastases and the manipulation of the EMT process [24,57]. In a study, Yang and colleagues observed that the expression of miR-149 is downregulated and was inversely correlated with the invasive potential of NSCLC cell lines. Further studies demonstrated that overexpression of miR-149 increased protein expression of E-cadherin and decreased protein expression of vimentin (involve in regulating EMT phenotype) in NSCLC cells. These findings indicate that miR-149 inhibits the EMT process, which is suggestive of its tumor-suppressive activity [24,57].

Importantly, the forkhead family of transcription factors such as Forkhead Box Protein M1 (FOXM1) has been identified to function as an oncogene and shown to promote tumor progression, invasion, and chemoresistance via its ability to target cell cycle proteins such as cyclin D1, and MMPs [23,24,57-63]. Notably, FOXM1 has also been found to be the target of several miRs in the NSCLC model. A study demonstrated that miR-149 efficiently inhibits the EMT process in human A549 and H1299 NSCLC cell lines by targeting FOXM1 [57]. These findings indicated that miR-149 restoration could be used as a possible therapeutic approach for NSCLC treatment. Another study by Zhao and colleagues have shown that miR-149 overexpression inhibits cell proliferation, migration, invasion, and induces apoptosis in human HCC827 and A549 cell lines. Importantly, miR-149 inhibition reverses these processes [61]. Further studies demonstrated that FOXM1 is a downstream target of miR-149 and downregulates cyclin D1 and MMP- 2 expression. The overexpression of miR-149 also inhibited the NSCLC tumor growth and metastasis in vivo [61]. Of significance, the clinical data suggested that miR-149 expression is downregulated in NSCLC patients, and was also correlated with patients' overall survival rate [61]. Together this evidence suggested that miR-149 functions as a potential tumor suppressor, and thus can be considered as a target to assess lung cancer progression, invasion, and metastasis.

Multiple studies have also reported that miR-149 effects are modulated by long non-coding RNAs (lncRNAs). LncRNAs consist of 200 nucleotides and are implicated in regulating gene expression at the transcriptional level by recruiting chromatin-modifying complexes, and at the posttranscriptional level through interacting 
Table 1: Summary of studies indicating miR-149 as a promising therapeutic target.

\begin{tabular}{|c|c|c|c|c|c|}
\hline No. & Research tools & $\begin{array}{l}\text { miR-149 expression } \\
\text { level }\end{array}$ & Targets & Findings & Refs. \\
\hline 1 & NSCLC cell lines - A549,Calu3, Calu1, H1299 & Downregulated & EMT process and FOXM1 & Functions as a suppressor of EMT & 57 \\
\hline 2 & $\begin{array}{l}\text { NSCLC patients, cell line - 293T, BEAS-2B, A549, } \\
\text { H1299, H1975, HCC827 and in vivo }\end{array}$ & Downregulated & FOXM1 & $\begin{array}{l}\text { Attenuates lung cancer growth and } \\
\text { metastasis }\end{array}$ & 61 \\
\hline 3 & $\begin{array}{l}\text { NSCLC patients, cell line - BEAS-2B, A549, } \\
\text { H1299, H1563, SKMES1, H1437, H2023 and in } \\
\text { vivo }\end{array}$ & Downregulated & $\begin{array}{l}\text { IncRNA HNF1A-AS1 and } \\
\text { Cdk6 }\end{array}$ & $\begin{array}{l}\text { Modulate lung cancer growth by } \\
\text { sponging miR-149 effects }\end{array}$ & 65 \\
\hline 4 & $\begin{array}{l}\text { NSCLC patients, cell lines - 16HBE, H460, SK- } \\
\text { MES-1, SPC-A-1, H1299 }\end{array}$ & Downregulated & IncRNA PCAT-1 and LRIG2 & $\begin{array}{l}\text { Augments cancer growth by sponging } \\
\text { the miR-149 effects }\end{array}$ & 64 \\
\hline 5 & $\begin{array}{l}\text { NSCLC patients, cell lines - 16HBE, A549, H1299, } \\
\text { H460 }\end{array}$ & Upregulated & $\begin{array}{l}\text { IncRNA BCYRN1, PKM2 and } \\
\text { glycolysis }\end{array}$ & $\begin{array}{l}\text { Promotes tumor progression by } \\
\text { regulating miR-149 }\end{array}$ & 68 \\
\hline 6 & $\begin{array}{l}\text { NSCLC patients, cell lines - BEAS-2B, H838, } \\
\mathrm{H} 1975, \mathrm{H} 157, \mathrm{H} 358, \mathrm{H} 1299 \text { and in vivo }\end{array}$ & Upregulated & $\begin{array}{l}\text { LINC00472, KLLN and p53 } \\
\text { signaling pathway }\end{array}$ & $\begin{array}{l}\text { IncRNA downregulate miR-149 effect } \\
\text { and suppresses tumor growth }\end{array}$ & 66 \\
\hline
\end{tabular}

Table 2: Summary of studies suggesting miR-149 as a potential biomarker.

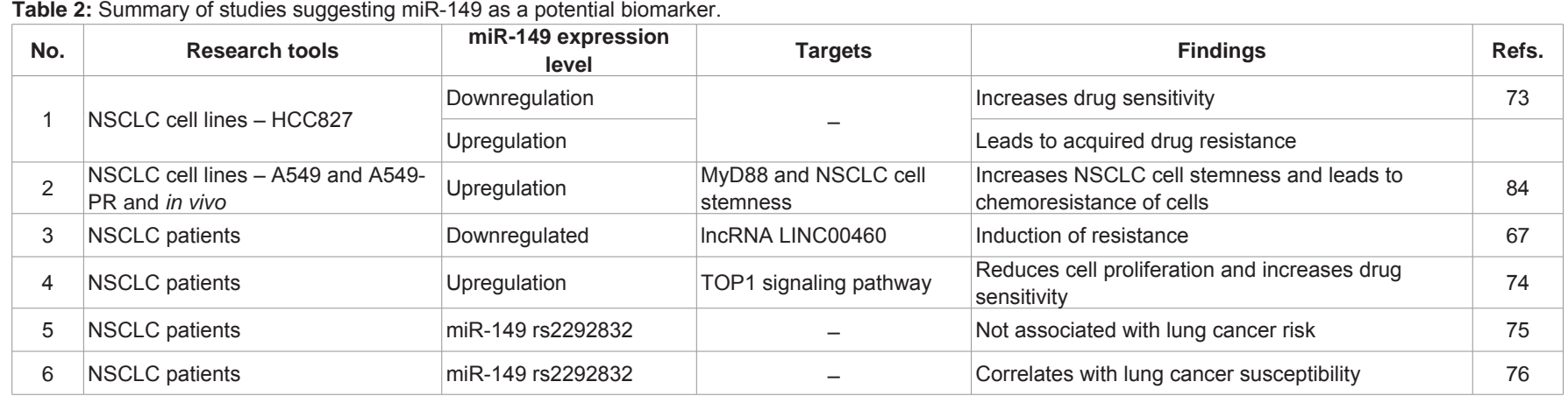

with miRs, mRNAs, and proteins [64-72]. While lncRNAs are unable to translate the protein [64-69], they play critical roles in cancer by dysregulating miRs function (66-72). To that end, a study by Liu et al., have demonstrated that IncRNA Hepatocyte Nuclear Factor-1 homeobox A (HNF1A)-antisense RNA 1 (AS1) (i.e., lncRNA HNF1A-AS1) directly binds to miR-149 and inhibits its expression via regulating cyclin-dependent kinase 6 (Cdk6) [65]. These findings indicate that lncRNA HNF1A-AS1 exerts oncogenic effect through its sponging effect on miR-149 in favoring NSCLC progression [65]. Along similar lines, another report has shown that lncRNA prostate cancer-associated ncRNA transcripts 1 (i.e., PCAT-1) mediate its effects by regulating miR-149 expression via leucine-rich repeats and immunoglobulin-like domains 2 (LRIG2) to modulate lung cancer progression [64].

Given that aerobic glycolysis converts glucose into pyruvate, which is then oxidized into lactate, resulting in the destruction of the ECM, and favoring cancer cells in acquiring the metastatic potential that leads to the augmentation of cell proliferation, and apoptotic resistance [73-86]. Studies have also hypothesized that lncRNAs may target the glycolysis process in cancer and found that lncRNA brain cytoplasmic RNA 1 (i.e., BcYrn1) regulates glycolysis in NSCLC [68]. This fact was also supported by studies demonstrating that miR-149 expression was significantly downregulated in A549 cells following lncRNA BcYrn1 overexpression via targeting Pyruvate Kinase M2 (PKM2) (68). In contrast, another study performed by Zou and colleagues have demonstrated that overexpression of long intergenic non-protein-coding RNA 472 (i.e., LINC00472) inhibits NSCLC growth via killin (KLLN)/p53 signaling by targeting miR-149 [66]. This evidence suggested that lncRNAs can modulate the tumorsuppressive action of miR-149 in promoting the growth of NSCLC. Thus, miR-149 could be targeted to circumvent these lncRNAs- induced effects in lung cancer. The studies suggesting miR-149 as a promising therapeutic target are summarized in Table 1.

\section{miR-149 as a potential biomarker}

In addition to the miR-149 potential to be used as a therapeutic target, multiple studies have also supported its applicability as a biomarker because chemoresistance remains an exciting and challenging area of therapeutic invention. Given that the induction of drug resistance could either be intrinsic or acquired and developed during the course of the treatment or post-treatment via various mechanisms, several studies have shown that miR-149 plays an important role in modulating the drug sensitivity as well as chemoresistance $[31,41]$. In the NSCLC model, an inhibitor of miR149 has been shown to increase the sensitivity of tyrosine kinases (i.e., gefitinib drug) whereas overexpression of miR-149 was found to result in acquired drug resistance [73]. As Cancer Stem Cells (CSCs) play critical roles in drug resistance, agents to target CSCs have been postulated to increase the drug sensitivity through modulating miRs against NSCLC [84]. In a study, Chen and colleagues demonstrated that the stemness of NSCLC cells is related to the levels of miR149 expression and drug resistance. The authors demonstrated that miR-149 overexpression can induce stemness in A549 cells whereas its inhibition can reduce the stemness of paclitaxel-resistant A549 cells. However, ursolic acid possessing anticancer effect was found to reduce the stemness of A549 resistant cells by its ability to increase miR-149 expression that overcomes the resistance of NSCLC cells via Myeloid differentiation primary response 88 (MyD88) [84]. Of importance, studies by Nakano and colleagues have shown that overexpression of long intergenic non-protein-coding RNA 460 (i.e., LINC00460) was associated with poor responses to osimertinib, gefitinib, and erlotinib treatments, and resulted in the induction of resistance mediated by decreasing miR-149 expression [67]. Taken 
together, these studies indicate that miR-149 can be considered as a potential biomarker for predicting the sensitivity or drug-resistance of lung cancer to therapeutic agents.

Of significance, several studies have discovered a correlation between Single Nucleotide Polymorphisms (SNPs) or mutations in miRs and the risk or susceptibility to lung cancer. SNPs represent an alternative type of genetic variation, which was suggestive to be associated with stimulating the risks and/or outcomes of human diseases. SNPs in pre- miRNAs have been shown to alter miRs expression that could influence the prognosis of lung cancer [7483]. Studies by Lingzi and colleagues conducted a prospective cohort study in the Chinese female population and found that NSCLC patients having a $\mathrm{C}$ allele expresses a high level of miR-149, which resulted in decreased cell proliferation and increased drug sensitivity via targeting topoisomerases 1 (TOP1) pathway [74]. These findings indicated that polymorphisms in miR-149 may be associated with the overall survival of NSCLC patients [74]. However, a case- control study conducted by $\mathrm{Li}$ and colleagues has reported that miR-149 rs2292832 polymorphism is not associated with lung cancer risk [75]. In contrast, another report by Fang and colleagues indicated that miR-149 rs71428439 polymorphism correlates with lung cancer susceptibility, and thus, may be beneficial in predicting the risk of occurrence of lung cancer [76]. Altogether these data suggest that genetic variations in miR-149 can be considered as a potential biomarker in the diagnosis or prognosis of lung cancer. The studies indicating the applicability of miR-149 as a potential biomarker are summarized in Table 2.

\section{Conclusion}

While several studies have suggested the current applicability of miR-149 in assessing tumor progression, invasion, metastasis, etc., using in vitro and in vivo model systems, as well as human samples, blinded case-controlled studies in diverse settings of lung cancer patients, are needed to confirm its implication as a therapeutic target or a biomarker. Moreover, the potential of miR-149 in predicting treatment outcomes is warranted.

\section{References}

1. Siegel RL, Miller KD, Jemal A. Cancer statistics, 2019. ca cancer j clin. 2019; 69: 7-34

2. National Cancer Institute.

3. Miller KD, Nogueira L, Mariotto AB, Rowland JH, Yabroff KR, Alfano CM, et al. Cancer treatment and survivorship statistics, 2019. CA: A Cancer Journa for Clinicians. 2019;69(5):363-85

4. Cancer in America - Most Common Cancers.

5. Cancer Facts \& Figures 2018.

6. Annual Report to the Nation 2020.

7. LSUHSC School of Medicine.

8. Lung Cancer Risk Factors: Smoking \& Lung Cancer.

9. American Lung Association

10. Centers for Disease Control and Prevention. Centers for Disease Control and Prevention; 2019.

11. Bender E. Epidemiology: The dominant malignancy. Nature. 2014; 513: S2S3.

12. Lemjabbar-Alaoui $\mathrm{H}$, Hassan OUI, Yang Y-W, Buchanan P. Lung cancer:
Biology and treatment options. Biochimica et Biophysica Acta (BBA) Reviews on Cancer. Elsevier; 2015; 1856: 189-210.

13. Dela Cruz CS, Tanoue LT, Matthay RA. Lung cancer: epidemiology, etiology, and prevention. Clinics in chest medicine. U.S. National Library of Medicine; 2011; 32: 605-644.

14. Lung Cancer: Diagnosis, Treatment Principles, and Screening.

15. Miller YE. Pathogenesis of Lung Cancer. American Journal of Respiratory Cell and Molecular Biology. 2005;33(3):216-23.

16. Bhaskaran M, Mohan M. MicroRNAs. Veterinary Pathology. 2013; 51: 759774.

17. Adams BD, Kasinski AL, Slack FJ. Aberrant Regulation and Function of MicroRNAs in Cancer. Current Biology. 2014; 24: R762-R776.

18. Davis BN, Hata A. Mechanisms of control of microRNA biogenesis. Journal of Biochemistry. 2010; 148: 381-392.

19. Catalanotto C, Cogoni C, Zardo G. MicroRNA in Control of Gene Expression: An Overview of Nuclear Functions. International Journal of Molecular Sciences. 2016; 17: 1712

20. Tan W, Liu B, Qu S, Liang G, Luo W, Gong C. MicroRNAs and cancer: Key paradigms in molecular therapy (Review). Oncology Letters. 2018; 15: 27352742.

21. Ying S-Y, Chang DC, Miller JD, Lin S-L. The MicroRNA: Overview of the RNA Gene That Modulates Gene Functions. MicroRNA Protocols. 2006; 342 : $1-18$

22. Noureddine L, Hajarnis S, Patel V. MicroRNAs and polycystic kidney disease. Drug Discovery Today: Disease Models. 2013; 10: e137-e1743.

23. Li M, Li J, Ding X, He M, Cheng SY. microRNA and cancer. AAPS J. 2010; 12: $309-17$

24. Wu K-L, Tsai Y-M, Lien C-T, Kuo P-L, Hung AJ-Y. The Roles of MicroRNA in Lung Cancer. International Journal of Molecular Sciences. 2019; 20: 1611.

25. Hanna J, Hossain GS, Kocerha J. The Potential for microRNA Therapeutics and Clinical Research. Front Genet. 2019 May 16; 10: 478.

26. Thyagarajan A, Shaban A, Sahu RP. MicroRNA-Directed Cancer Therapies: Implications in Melanoma Intervention. Journal of Pharmacology and Experimental Therapeutics. 2017; 364: 1-12.

27. Macha M, Seshacharyulu P, Krishn S, Pai P, Rachagani S, Jain M, et al. MicroRNAs (miRNAs) as Biomarker(s) for Prognosis and Diagnosis of Gastrointestinal (GI) Cancers. Current Pharmaceutical Design. 2014; 20 : 5287-97.

28. Zubor P, Kubatka P, Dankova Z, Gondova A, Kajo K, Hatok J, et al. miRNA in a multiomic context for diagnosis, treatment monitoring and personalized management of metastatic breast cancer. Future Oncology. 2018; 14: 1847 1867.

29. Melo SA, Esteller M. Dysregulation of microRNAs in cancer: Playing with fire. FEBS Letters. 2010; 585: 2087-2099.

30. miRBase.

31. He Y, Yu D, Zhu L, Zhong S, Zhao J, Tang J. miR-149 in Human Cancer: A Systemic Review. Journal of Cancer. 2018;9(2):375-88.

32. Tian P, Yan L. Inhibition of MicroRNA-149-5p Induces Apoptosis of Acute Myeloid Leukemia Cell Line THP-1 by Targeting Fas Ligand (FASLG). Medical Science Monitor. 2016; 22: 5116-23.

33. Fujii T, Shimada K, Tatsumi Y, Fujimoto K, Konishi N. Syndecan-1 responsive microRNA-126 and 149 regulate cell proliferation in prostate cancer. Biochemical and Biophysical Research Communications. 2015; 456: 183189.

34. Wang Y, Zheng X, Zhang Z, Zhou J, Zhao G, Yang J, et al. MicroRNA-149 Inhibits Proliferation and Cell Cycle Progression through the Targeting of ZBTB2 in Human Gastric Cancer. PLoS ONE. 2012; 7: 1-10.

35. Zhang Y, Guo X, Xiong L, Yu L, Li Z, Guo Q, et al. Comprehensive analysis 
of microRNA-regulated protein interaction network reveals the tumor suppressive role of microRNA-149 in human hepatocellular carcinoma via targeting AKT-mTOR pathway. Molecular Cancer. 2014; 13: 253.

36. Shen X, Li J, Liao W, Wang J, Chen H, Yao Y, et al. microRNA-149 targets caspase-2 in glioma progression. Oncotarget. 2016; 7: 26388-99.

37. Xu Y, Chen X, Lin L, Chen H, Yu S, Li D. MicroRNA-149 is associated with clinical outcome in human neuroblastoma and modulates cancer cell proliferation through Rap1 independent of MYCN amplification. Biochimie. 2017; 139: 1-8

38. Bischoff A, Huck B, Keller B, Strotbek M, Schmid S, Boerries M, et al. miR149 Functions as a Tumor Suppressor by Controlling Breast Epithelial Cell Migration and Invasion. Cancer Research. 2014; 74: 5256-65

39. Bischoff A, Bayerlová M, Strotbek M, Schmid S, Beissbarth T, Olayioye MA A global microRNA screen identifies regulators of the ErbB receptor signaling network. Cell Communication and Signaling. 2015; $13: 5$

40. Chan S-H, Huang W-C, Chang J-W, Chang K-J, Kuo W-H, Wang M-Y, et al MicroRNA-149 targets GIT1 to suppress integrin signaling and breast cancer metastasis. Oncogene. 2014; 33: 4496-4507.

41. Zhi $Y$, Zhou H, Mubalake A, Chen $Y$, Zhang B, Zhang $K$, et al. Regulation and functions of MicroRNA- 149 in human cancers. Cell Proliferation. 2018; 51: e12465.

42. Lin R-J, Lin Y-C, Yu AL. Abstract 2038: miR-149* induces apoptosis by inhibiting Akt1 and E2F1 in human cancer cells. Cellular and Molecular Biology. 2010; 49: 719-27.

43. Ye X, Chen X. miR-149-5p inhibits cell proliferation and invasion through targeting GIT1 in medullary thyroid carcinoma. Oncology Letters. 2018; 17 : $372-378$

44. Suzuki H, Maruyama R, Yamamoto E, Kai M. Epigenetic alteration and microRNA dysregulation in cancer. Frontiers in Genetics. 2013; 4: 258.

45. Sheervalilou R, Shirvaliloo S, Aval SF, Khamaneh AM, Sharifi A, Ansarin K, et al. A new insight on reciprocal relationship between microRNA expression and epigenetic modifications in human lung cancer. Tumor Biology. 2017; 39 101042831769503

46. Rahman MM, Brane AC, Tollefsbol TO. MicroRNAs and Epigenetics Strategies to Reverse Breast Cancer. Cells. 2019; 8: 1214.

47. Bosutti A, Zanconati F, Grassi G, Dapas B, Passamonti S, Scaggiante B Epigenetic and miRNAs Dysregulation in Prostate Cancer: The role of Nutraceuticals. Anti-Cancer Agents in Medicinal Chemistry. 2016; 16: 1385402

48. Dillekås H, Rogers MS, Straume O. Are $90 \%$ of deaths from cancer caused by metastases? Cancer Medicine. 2019; 8: 5574-6.

49. Guan X. Cancer metastases: challenges and opportunities. Acta Pharmaceutica Sinica B. 2015; 5: 402-418.

50. Du L, Pertsemlidis A. microRNAs and lung cancer: tumors and 22-mers. Cancer and Metastasis Reviews. 2010; 29: 109-122.

51. Paolillo, Schinelli. Extracellular Matrix Alterations in Metastatic Processes. International Journal of Molecular Sciences. 2019; 20: 4947

52. Bonnans C, Chou J, Werb Z. Remodelling the extracellular matrix in development and disease. Nature Reviews Molecular Cell Biology. 2014; 15 786-801.

53. Rupaimoole R, Calin GA, Lopez-Berestein G, Sood AK. miRNA Deregulation in Cancer Cells and the Tumor Microenvironment. Cancer Discovery. 2016 6: 235-246.

54. Xiao W, Wang X, Wang T, Xing J. Overexpression of BMP1 reflects poor prognosis in clear cell renal cell carcinoma. Cancer Gene Therapy. 2019 27: $330-340$

55. Winer A, Adams S, Mignatti P. Matrix Metalloproteinase Inhibitors in Cance Therapy: Turning Past Failures Into Future Successes. Molecular Cancer Therapeutics. 2018; 17:1147-55.

56. Clark E, Weaver A. A new role for cortactin in invadopodia: Regulation of protease secretion. European Journal of Cell Biology. 2008; 87: 581-90.

57. Ke Y, Zhao W, Xiong J, Cao R. miR-149 Inhibits Non-Small-Cell Lung Cancer Cells EMT by Targeting FOXM1. Biochemistry Research International. 2013; 2013: 1-8.

58. Kim I-M, Ackerson T, Ramakrishna S, Tretiakova M, Wang I-C, Kalin TV, et al. The Forkhead Box m1 Transcription Factor Stimulates the Proliferation of Tumor Cells during Development of Lung Cancer. Cancer Research. 2006; 66: 2153-61.

59. Kong F-F, Qu Z-Q, Yuan H-H, Wang J-Y, Zhao M, Guo Y-H, et al Overexpression of FOXM1 is associated with EMT and is a predictor of poo prognosis in non-small cell lung cancer. Oncology Reports. 2014; 31: 2660 2668.

60. Wang K, Zhu X, Zhang K, Zhu L, Zhou F. FoxM1 inhibition enhances chemosensitivity of docetaxel resistant A549 cells to docetaxel via activation of JNK/mitochondrial pathway. Acta Biochimica et Biophysica Sinica. 2016; 48: 804-809.

61. Zhao L, Liu L, Dong Z, Xiong J. miR-149 suppresses human non-small cel lung cancer growth and metastasis by inhibiting the FOXM1/cyclinD1/MMP2 axis. Oncology Reports. 2017; 38: 3522-3530.

62. Chen P-M, Wu T-C, Shieh S-H, Wu Y-H, Li M-C, Sheu G-T, et al. MnSOD Promotes Tumor Invasion via Upregulation of FoxM1-MMP2 Axis and Related with Poor Survival and Relapse in Lung Adenocarcinomas. Molecular Cancer Research. 2012; 11: 261-71.

63. Liu Y, Hock JM, Beneden RJV, Li X. Aberrant overexpression of FOXM1 transcription factor plays a critical role in lung carcinogenesis induced by low doses of arsenic. Molecular Carcinogenesis. 2012; 53: 380-391.

64. Li J, Li Y, Wang B, Ma Y, Chen P. LncRNA PCAT 1 promotes non-small cell lung cancer progression by regulating miR-149 5p/LRIG2 axis. Journal of Cellular Biochemistry. 2018; 120: 7725-7733

65. Liu L, Chen Y, Li Q, Duan P. IncRNA HNF1A AS1 modulates non-small cell lung cancer progression by targeting miR-149 5p/Cdk6. Journal of Cellular Biochemistry. 2019; 120: 18736-18750.

66. Zou A, Liu X, Mai Z, Zhang J, Liu Z, Huang Q, et al. LINC00472 Acts as a Tumor Suppressor in NSCLC through KLLN-Mediated p53-Signaling Pathway via MicroRNA-149-3p and MicroRNA-4270. Molecular Therapy Nucleic Acids. 2019; 17: 563-577

67. Nakano Y, Isobe K, Kobayashi H, Kaburaki K, Isshiki T, Sakamoto S, et al. Clinical importance of long non-coding RNA LINC00460 expression in EGFRmutant lung adenocarcinoma. International Journal of Oncology. 2019; 56; 243-257.

68. Lang N, Wang $\mathrm{C}$, Zhao J, Shi $\mathrm{F}$, Wu T, Cao $\mathrm{H}$. Long non-coding RNA BCYRN1 promotes glycolysis and tumor progression by regulating the miR149/PKM2 axis in non-small-cell lung cancer. Molecular Medicine Reports. 2020; 21: 1509-1516.

69. Roth A, Diederichs S. Long Noncoding RNAs in Lung Cancer. Current Topics in Microbiology and Immunology Long Non-coding RNAs in Human Disease. 2015; 394: 57-110.

70. Deng G, Sui G. Noncoding RNA in Oncogenesis: A New Era of Identifying Key Players. International Journal of Molecular Sciences. 2013; 14: 18319-

71. Dimitrova N. Genetic Variations of Long Noncoding RNAs in Cancer. Cancer and Noncoding RNAs. 2018; 289-308.

72. Renganathan A, Felley-Bosco E. Long Noncoding RNAs in Cancer and Therapeutic Potential. Advances in Experimental Medicine and Biology Long Non-Coding RNA Biology. 2017; 199-222.

73. Hu Y, Qin X, Yan D, Cao H, Zhou L, Fan F, et al. Genome-wide profiling of micro-RNA expression in gefitinib-resistant human lung adenocarcinoma using microarray for the identification of miR-149-5p modulation. Tumor Biology. 2017; 39:101042831769165.

74. Lingzi X, Zhihua Y, Xuelian L, Yangwu R, Haibo Z, Yuxia Z, et al. Genetic variants in microRNAs predict non-small cell lung cancer prognosis in 
Chinese female population in a prospective cohort study. Oncotarget. 2016 7: 83101-14

75. Li H, Ren Y, Xia L, Qu R, Kong L, Yin Z, et al. Association of MicroRNA-149 Polymorphism with Lung Cancer Risk in Chinese Non-Smoking Female: A Case-Control Study. Plos One. 2016; 11: 1-10

76. Fang C, Li X-P, Chen Y-X, Wu N-Y, Yin J-Y, Zhang W, et al. Functional miRNA variants affect lung cancer susceptibility and platinum-based chemotherapy response. Journal of Thoracic Disease. 2018; 10: 3329-40.

77. Xu L, Tang W. The associations of nucleotide polymorphisms in mir-196a2, mir-146a, mir-149 with lung cancer risk. Cancer Biomarkers. 2015; 15: 57-63.

78. Vinci S, Gelmini S, Pratesi N, Conti S, Malentacchi F, Simi L, et al. Genetic variants in miR-146a, miR- 149, miR-196a2, miR-499 and their influence on relative expression in lung cancers. Clinical Chemistry and Laboratory Medicine (CCLM). 2011; 49: 2073-2080.

79. Fan X, Wu Z. Effects of four single nucleotide polymorphisms in microRNAcoding genes on lung cancer risk. Tumor Biology. 2014; 35: 10815-24.

80. Chen Z, Xu L, Ye X, Shen S, Li Z, Niu X, et al. Polymorphisms of microRNA Sequences or Binding Sites and Lung Cancer: A Meta-Analysis and Systematic Review. PLoS ONE. 2013; 8: e61008.
81. Zhang J, Liu Y-F, Gan Y. Lack of association between miR-149 C>T polymorphism and cancer susceptibility: a meta-analysis based on 4,677 cases and 4,830 controls. Molecular Biology Reports. 2012; 39: 8749-8753.

82. Xia L, Ren Y, Fang X, Yin Z, Li X, Wu W, et al. Prognostic Role of Common MicroRNA Polymorphisms in Cancers: Evidence from a Meta-Analysis. PLoS ONE. 2014; 9: e106799.

83. Hong MJ, Choi YY, Jang J-A, Jung H-J, Lee SY, Lee WK, et al. Association between Genetic Variants in Pre-MicroRNAs and Survival of Early-Stage NSCLC. Journal of Thoracic Oncology. 2013; 8: 703-710.

84. Kong X-Z, Hu S-S, Sun Z, Zuo L-H, Kang J, Zhu Z-F, et al. Regulation of aerobic glycolysis by long non- coding RNAs in cancer. Biochemical and Biophysical Research Communications. 2016; 479: 28-32.

85. Lim S-O, Li C-W, Xia W, Lee H-H, Chang S-S, Shen J, et al. EGFR Signaling Enhances Aerobic Glycolysis in Triple-Negative Breast Cancer Cells to Promote Tumor Growth and Immune Escape. Cancer Research. 2016; 76 : 1284-1296

86. Goncalves MD, Cantley LC. A Glycolysis Outsider Steps into the Cancer Spotlight. Cell Metabolism. 2018; 28: 3-4. 